\title{
Penile Strangulation: A Novel Surgical Procedure without Cutting Equipment
}

\author{
Young Gon Kim, Yu Seob Shin, Jae Hyung You \\ Department of Urology, Jeonbuk National University Medical School, and Research Institute of Clinical Medicine of Jeonbuk National \\ University-Biomedical Research Institute of Jeonbuk National University Hospital, Jeonju, Korea
}

Strangulation of the penis is a rare condition that usually results from the placement of various constricting devices to enhance sexual stimulation. Such a condition requires emergency treatment to prevent penile ischemia, necrosis, urethral injury, and erectile dysfunction. This paper reports the case of a 51-year-old man who was referred for penile pain and swelling following the self-placement of an industrial metallic ring at the base of the penis. The metallic ring was removed safely without using specialized equipment.

Keywords: Penis; Penile diseases; Foreign bodies

Copyright (c) 2020, Korean Association of Urogenital Tract Infection and Inflammation. All rights reserved. (1) (\$) This is an open access article distributed under the terms of the Creative Commons Attribution Non-Commercial License (http://creativecommons.org/licenses/by-nc/4.0) which permits unrestricted non-commercial use, distribution, and reproduction in any medium, provided the original work is properly cited.
Received: 14 January, 2020

Revised: 29 January, 2020

Accepted: 29 January, 2020

\author{
Correspondence to: Jae Hyung You \\ (iD) https://orcid.org/0000-0002-1510-6372 \\ Department of Urology, Jeonbuk National Univer- \\ sity Hospital, 20 Geonji-ro, Deokjin-gu, Jeonju \\ 54907, Korea \\ Tel: +82-63-250-1560, Fax: +82-63-250-1564 \\ E-mail: yjh21111@hanmail.net
}

Strangulation of the penis is a rare condition that is usually caused by the placement of various constricting devices to enhance sexual stimulation. This condition requires emergency treatment to prevent penile ischemia, necrosis, urethral injury, and erectile dysfunction. Non-metallic and thin metallic devices can be removed easily by cutting, whereas thick metallic devices require a specialized saw or electrical cutting machine, which are unfamiliar and dangerous equipment for urologists. This paper reports the case of a 51year-old man who was referred for penile pain and swelling following the self-placement of an industrial metallic ring at the base of the penis four days before presentation to the hospital. The metallic ring was removed safely without using specialized equipment.

\section{CASE REPORT}

A 51-year-old man was referred for penile pain and swelling following the self-placement of an industrial metallic ring at the base of his penis four days earlier. His medical history was unremarkable. A physical examination revealed extensive edema of the penis distal to the metallic ring. The ring had an internal diameter of $30 \mathrm{~mm}$, an external diameter of $55 \mathrm{~mm}$, and a thickness of $25 \mathrm{~mm}$ (Fig. 1A). At the hospital,
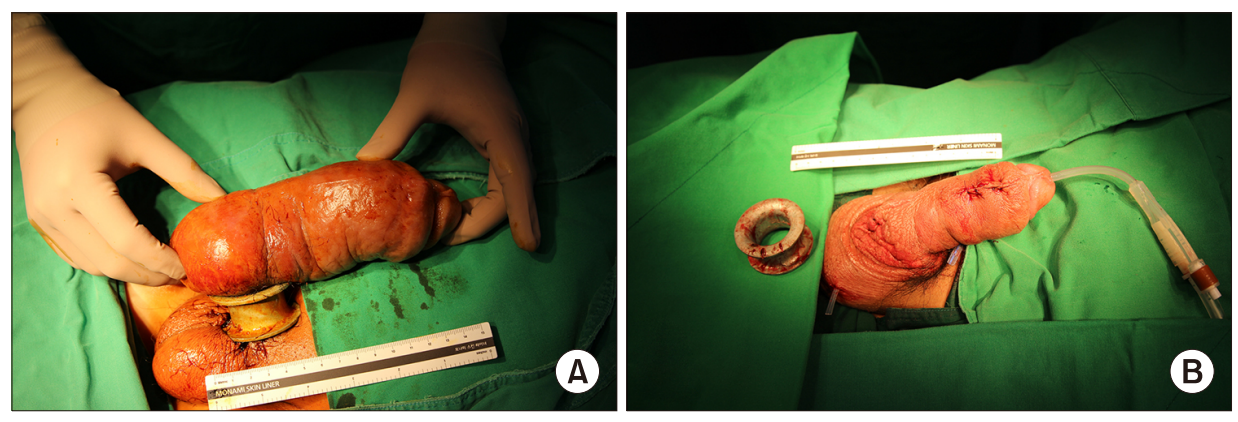

Fig. 1. (A) Thick metallic ring is strangling the penis. Note the extensive penile edema. (B) The thick metallic ring was removed successfully and safely. 

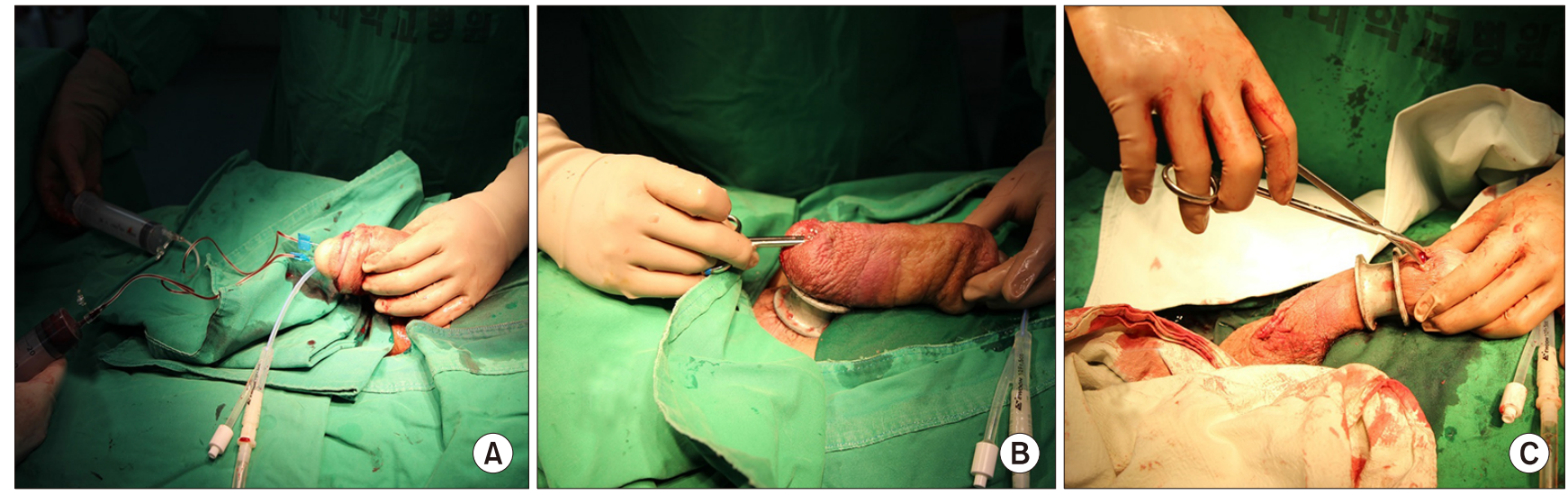

Fig. 2. Surgical procedure. (A) Aspiration the blood from the erectile tissue. (B, C) Incision and edematous fluid drainage at the proximal and distal penis.

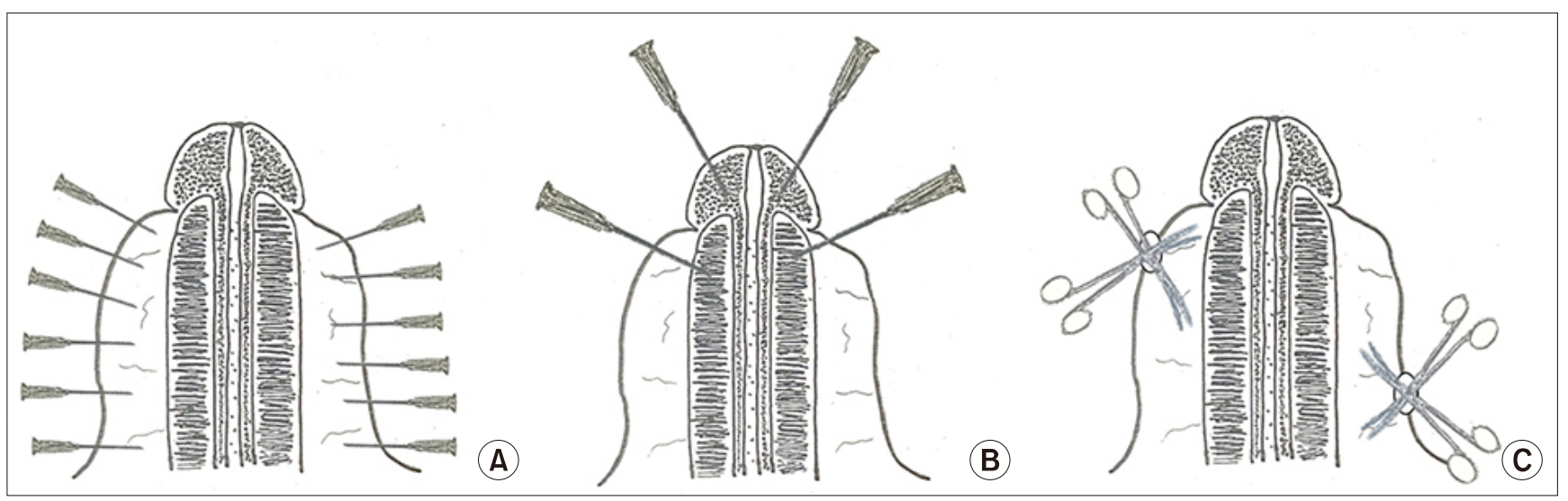

Fig. 3. Schematic diagram of the surgical procedure. (A) Multiple needle pricks on the penile skin. (B) Aspiration the blood from the erectile tissue. (C) Incision and edematous fluid drainage.

there was no special equipment to cut the ring, which was quite hard. After explaining the procedure to the patient, a decision was made to remove the metallic ring by resolving the edema of the penile skin and aspirating the blood from the erectile tissue.

Under spinal anesthesia, the patient was placed on the table in the supine position. The operating field was prepared using alcohol and betadine. In particular, a thin cotton swab was used to wipe the area between the ring and skin as much as possible. Third-generation cephalosporins and aminoglycosides were administered as prophylactic antibiotics. As the first step, multiple needle pricks were made on the penile skin using an 18-gauge needle to reduce the edema. In the second step, each of the two 24-gauge needles was placed in the corpus spongiosum and corpus cavernosum to remove blood from the erectile tissue. Even after the above two steps were carried out, the metallic ring could not be removed. Finally, approximately $1-\mathrm{cm}$ incisions were made in the distal and proximal parts of the penis. To break up the edematous fluid loculations, blunt dissection of the edematous skin was performed using blunt scissors through the incision sites. Gentle pressure was then applied to the penis by hand, and the serosanguineous fluid was drained through the incision sites (Fig. 2, 3). After this process, the metallic ring was removed successfully. A silicone drain was placed in the proximal and distal parts of the penis, and the incision sites were repaired using 3-0 Nylon sutures (Fig. 1B). Three months later, the patient had no problems concerning the penile skin or erectile function.

\section{DISCUSSION}

Penile strangulation is a rare condition that requires immediate treatment. To date, penile constriction rings have been removed using cutting equipment in almost all cases. On the other hand, such cutting equipment is unfamiliar 
and dangerous for urologists [1]. A small number of cases reported ring removal using the Dundee technique with blood aspiration from the erectile tissue [2-4]. The two techniques described above were performed, but the ring could not be removed. To remove the large amount of subcutaneous edema remaining, two incisions were made on the penile skin, and the edematous fluid was drained. To the best of the author's knowledge, drainage of the edematous fluid after an incision of the penile skin in penile strangulation has not been attempted previously. After the incision and drainage technique, the edema of the penile skin was resolved effectively, after which the ring was removed quickly.

The metallic ring was removed safely and successfully using a unique surgical procedure. Because penile strangulation is a rare situation, this surgical procedure has only been performed once. It is hoped that other urologists can performed this surgical procedure in the future and that the safety and effectiveness of the procedure can be verified.

\section{CONFLICT OF INTEREST}

No potential conflict of interest relevant to this article was reported.

\section{AUTHOR CONTRIBUTIONS}

Y.G.K. participated in data collection and wrote the manuscript. Y.G.K., Y.S.S., and J.H.Y. participated in the study design and performed the statistical analysis. J.H.Y. participated in the study design and coordination and helped to draft the manuscript. All authors read and approved the final manuscript.

\section{ORCID}

Young Gon Kim, https://orcid.org/0000-0001-7522-3266 Yu Seob Shin, https://orcid.org/0000-0002-1126-3821

Jae Hyung You, https://orcid.org/0000-0002-1510-6372

\section{REFERENCES}

1. Horstmann M, Mattsson B, Padevit C, Gloyer M, Hotz T, John $\mathrm{H}$. Successful removal of a $3.6-\mathrm{cm}$ long metal band used as a penile constriction ring. J Sex Med 2010;7:3798-801.

2. Talib RA, Canguven O, Al Ansari A, Shamsodini A. Treatment of penile strangulation by the rotating saw and 4-needle aspiration method: two case reports. Arch Ital Urol Androl 2014;86:138-9.

3. Liu GM, Sun G, Ma HS. Extrication of penile entrapment in a polyethylene terephthalate (PET) bottle: a technique of suture traction and Dundee and literature review. Int Urol Nephrol 2012;44:1335-40.

4. Wu X, Batra R, Al-Akraa M, Seneviratne LN. Penoscrotal entrapment: a safe, innovative technique for removing metal constricting devices. BMJ Case Rep 2012;2012:bcr2012006466. 